\title{
Geheimdienste gefährden massiv den Datenverkehr zwischen Deutschland und außereuropäischen Staaten
}

Angesichts der Berichte über die umfassenden und anlasslosen Überwachungsmaßnahmen ausländischer Geheimdienste, insbesondere der US-amerikanischen National Security Agency (NSA), weist die Konferenz der Datenschutzbeauftragten des Bundes und der Länder mit dieser Pressemitteilung vom 24.07.2013 auf die Befugnisse hin, die den Aufsichtsbehörden beim internationalen Datenverkehr zwischen Unternehmen in Deutschland und Drittstaaten nach dem Bundesdatenschutzgesetz und der europäischen Datenschutzrichtlinie bereits jetzt zustehen.

Die Europäische Kommission hat in mehreren Entscheidungen Grundsätze des „sicheren Hafens“ („Safe Harbor“) zum Datentransfer in die USA (2000) und Standardvertragsklauseln zum Datentransfer auch in andere Drittstaaten (2004 und 2010) festgelegt. Die Beachtung dieser Vorgaben soll gewährleisten, dass personenbezogene Daten, die in die USA oder andere Drittstaaten übermittelt werden, dort einem angemessenen Datenschutzniveau unterliegen. Allerdings hat die Kommission stets betont, dass die nationalen Aufsichtsbehörden die Datenübermittlung dorthin aussetzen können, wenn eine „hohe Wahrscheinlichkeit“ besteht, dass die Safe-Harbor-Grundsätze oder Standardvertragsklauseln verletzt sind.

Dieser Fall ist jetzt eingetreten. Die Grundsätze in den Kommissionsentscheidungen sind mit hoher Wahrscheinlichkeit verletzt, weil die NSA und andere ausländische Geheimdienste nach den gegenwärtigen Erkenntnissen umfassend und anlasslos ohne Einhaltung der Grundsätze der Erforderlichkeit, Verhältnismäßigkeit und Zweckbindung auf personenbezogene Daten zugreifen, die von Unternehmen in Deutschland an Stellen in den USA übermittelt werden. Zwar enthält die Safe-Harbor-Vereinbarung eine Regelung, die die Geltung der Grundsätze des „sicheren Hafens" begrenzt, sofern es die nationale Sicherheit erfordert oder Gesetze solche Ermächtigungen vorsehen. Im Hinblick auf das Ziel eines wirksamen Schutzes der Privatsphäre soll jedoch von diesen Eingriffsbefugnissen nur im Rahmen des tatsächlich Erforderlichen und nicht exzessiv Gebrauch gemacht werden. Ein umfassender und anlassloser Zugriff auf personenbezogene Daten kann daher durch Erwägungen zur nationalen Sicherheit in einer demokratischen Gesellschaft nicht gerechtfertigt werden. Auch bei Datenübermittlungen in die USA aufgrund der Standardverträge muss der Datenimporteur zusichern, dass seines Wissens in seinem Land keine Rechtsvorschriften bestehen, die die Garantien aus den Klauseln in gravierender Weise beeinträchtigen. Eine solche Generalermächtigung scheint in den USA zu bestehen; denn nur so lässt sich erklären, dass der US-amerikanische Geheimdienst auf personenbezogene Daten, die aufgrund der Standardverträge übermittelt werden, mit hoher Wahrscheinlichkeit routinemäßig zugreift.

Deshalb fordert die Konferenz die Bundesregierung auf, plausibel darzulegen, dass der unbeschränkte Zugriff ausländischer Nachrichtendienste auf die personenbezogenen Daten der Menschen in Deutschland effektiv im Sinne der genannten Grundsätze begrenzt wird. Bevor dies nicht sichergestellt ist, werden die Aufsichtsbehörden für den Datenschutz keine neuen Genehmigungen für die Datenübermittlung in Drittstaaten (z. B. auch zur Nutzung bestimmter Cloud-Dienste) erteilen und prüfen, ob solche Datenübermittlungen auf der Grundlage des Safe-Harbor-Abkommens und der Standardvertragsklauseln auszusetzen sind.

Schließlich fordert die Konferenz die Europäische Kommission auf, ihre Entscheidungen zu Safe Harbor und zu den Standardverträgen vor dem Hintergrund der exzessiven Überwachungstätigkeit ausländischer Geheimdienste bis auf weiteres zu suspendieren.

Die diesjährige Vorsitzende der Konferenz der Datenschutzbeauftragten des Bundes und der Länder, Dr. Imke Sommer, sagte hierzu: „Wirtschaftsunternehmen, die personenbezogene Daten in die USA übermitteln, tragen für diese Daten die Verantwortung. Wie alle Menschen in Deutschland müssen auch sie deshalb ein Interesse daran haben, dass personenbezogene Datenflüsse von Geheimdiensten nicht im großen Stil anlasslos überwacht werden." 